\section{RMD Open}

Rheumatic \&

Musculoskeletal Diseases

\title{
Upadacitinib in patients with psoriatic arthritis and an inadequate response to non-biological therapy: 56-week data from the phase 3 SELECT-PsA 1 study
}

To cite: McInnes IB, Kato K Magrey M, et al. Upadacitinib in patients with psoriatic arthritis and an inadequate response to non-biological therapy: 56 week data from the phase 3 SELECT-PSA 1 study. RMD Open 2021;7:e001838. doi:10.1136/ rmdopen-2021-001838

- Additional supplemental material is published online only. To view, please visit the journal online (http://dx.doi.org/10. 1136/rmdopen-2021-001838).

Received 22 July 2021 Accepted 9 September 2021

Check for updates

(C) Author(s) (or their employer(s)) 2021. Re-use permitted under CC BY-NC. No commercial re-use. See rights and permissions. Published by BMJ.

For numbered affiliations see end of article.

Correspondence to Professor lain B McInnes; iain.mcinnes@glasgow.ac.uk

\section{ABSTRACT}

Background In SELECT-PSA 1, a randomised double-blind phase 3 study, upadacitinib $15 \mathrm{mg}$ and $30 \mathrm{mg}$ were superior to placebo and non-inferior to adalimumab in $\geq 20 \%$ improvement in American College of Rheumatology (ACR) criteria at 12 weeks in patients with psoriatic arthritis (PSA). Here, we report 56-week efficacy and safety in patients from SELECT-PSA 1. Methods Patients received upadacitinib $15 \mathrm{mg}$ or $30 \mathrm{mg}$ once daily, adalimumab $40 \mathrm{mg}$ every other week for 56 weeks or placebo through week 24 switched thereafter to upadacitinib $15 \mathrm{mg}$ or $30 \mathrm{mg}$ until week 56 . Efficacy endpoints included the proportion of patients achieving $\geq 20 \% / 50 \% / 70 \%$ improvement in ACR criteria (ACR20/50/70), $\geq 75 \% / 90 \% / 100 \%$ improvement in Psoriasis Area and Severity Index (PASI75/90/100), minimal disease activity (MDA) and change from baseline in modified total Sharp/van der Heijde Score. Treatment-emergent adverse events per 100 patient years (PY) were summarised.

Results Consistent with results through week 24, ACR20/50/70, PASI75/90/100 and MDA responses were maintained with upadacitinib through week 56 and were generally numerically higher than with adalimumab; inhibition of radiographic progression was also maintained. Patients who switched from placebo to upadacitinib exhibited comparable improvements at week 56 as patients originally randomised to upadacitinib. The rates of serious adverse events were 9.1 events/100 PY with upadacitinib $15 \mathrm{mg}$ and 12.3 events/100 PY with upadacitinib $30 \mathrm{mg}$. Two deaths were reported in each of the upadacitinib groups.

Conclusion Efficacy across various domains of PsA were maintained with upadacitinib $15 \mathrm{mg}$ and $30 \mathrm{mg}$ through week 56 with no new safety signals observed.

\section{INTRODUCTION}

The treatment goal for psoriatic arthritis (PsA) is to maximise patient outcomes by controlling inflammation and preventing irreversible joint damage and disability. ${ }^{12}$ Treat-to-target strategies optimise treatment until the desired management goal, such as

\section{Key messages}

What is already known about this subject?

- Despite the availability of biological diseasemodifying antirheumatic drugs (DMARDs) in psoriatic arthritis (PsA), only a small percentage of patients achieve low disease activity; therefore, additional treatment options are needed.

- In the SELECT-PSA 1 study, through 24 weeks, once daily upadacitinib $15 \mathrm{mg}$ and $30 \mathrm{mg}$ demonstrated improvements in clinical manifestations of PsA including musculoskeletal symptoms (peripheral arthritis, enthesitis and dactylitis), psoriasis, physical function, pain, fatigue and quality of life (QoL), as well as inhibition of radiographic progression in patients with PsA and inadequate response or intolerance to $\geq 1$ non-biological DMARD. Additionally, the results demonstrated non-inferiority of both upadacitinib doses and superiority of upadacitinib $30 \mathrm{mg}$ versus adalimumab in American College of Rheumatology (ACR) 20 response at week 12.

minimal disease activity (MDA), is achieved and maintained. Such an approach can improve long-term joint and skin outcomes and quality of life (QoL). ${ }^{34}$ Although multiple therapeutic choices are available, additional options are needed as under one third of patients achieve MDA in most placebocontrolled trials. ${ }^{5-10}$ Upadacitinib is an oral, reversible Janus kinase inhibitor approved for the treatment of rheumatoid arthritis (RA), PsA and ankylosing spondylitis in the EU. ${ }^{11-15}$ The results of SELECT-PsA 1 through 24 weeks demonstrated that once daily (QD) upadacitinib $15 \mathrm{mg}$ and $30 \mathrm{mg}$ were more efficacious than placebo for clinical manifestations of PsA including musculoskeletal symptoms (peripheral arthritis, enthesitis and 


\section{Key messages}

What does this study add?

- Consistent with responses through week 24, between weeks 24 and 56 of the SELECT-PSA 1 study, responses for clinical manifestations of PSA including musculoskeletal symptoms (peripheral arthritis, enthesitis, dactylitis and spondylitis), psoriasis, physical function, pain, fatigue and QoL, as well as inhibition of radiographic progression were increased or maintained with upadacitinib $15 \mathrm{mg}$ and $30 \mathrm{mg}$. ACR20/50/70 and Psoriatic Arthritis Response Criteria responses were numerically greater with upadacitinib $15 \mathrm{mg}$ and $30 \mathrm{mg}$ versus adalimumab. Safety data through week 56 were also consistent with week 24 and the upadacitinib rheumatoid arthritis trials and did not show any new safety signal.

- Efficacy results in patients who switched from placebo to upadacitinib were comparable to those observed in patients originally randomised to upadacitinib.

How might this impact on clinical practice or future developments?

- 56-week efficacy data across all domains of PsA support the benefits of continued upadacitinib therapy in patients with PSA. Safety at week 56 was comparable to findings through week 24 .

dactylitis), psoriasis, physical function, pain, fatigue and QoL, as well as inhibiting radiographic progression in patients with PsA and inadequate response (IR) or intolerance to $\geq 1$ non-biological disease-modifying antirheumatic drug (DMARD). ${ }^{16}$ At week 24, greater improvements (nominal $\mathrm{p} \leq 0.05$ ) were observed with upadacitinib $15 \mathrm{mg}$ and $30 \mathrm{mg}$ versus adalimumab in 20\%/50\%/70\% improvement in American College of Rheumatology (ACR) criteria (ACR20/50/70 responses). Here, we report safety and efficacy of upadacitinib versus adalimumab over 56 weeks from SELECT-PsA 1.

\section{PATIENTS AND METHODS}

\section{Patients and study design}

Inclusion criteria have been described previously. ${ }^{16}$ Briefly, patients in SELECT-PsA 1 (NCT03104400) were $\geq 18$ years of age with active PsA and IR or intolerance to $\geq 1$ non-biological DMARD. Patients were blindly randomised to upadacitinib $15 \mathrm{mg}$ or $30 \mathrm{mg}$ QD, placebo or adalimumab $40 \mathrm{mg}$ every other week. At week 24, all placebo patients switched to upadacitinib $15 \mathrm{mg}$ or $30 \mathrm{mg}$. Blinding was maintained to the sites until all patients reached the week 56 visit.

Stable treatment of non-steroidal anti-inflammatory drugs, corticosteroids and $\leq 2$ non-biological DMARDs was permitted through week 36 but not required. However, after the week 16 visit had been completed, patients who qualified for rescue therapy were permitted to add or modify background therapy. After week 36, initiation or change in background PsA medication(s) was permitted for all patients. From week 36, all patients not achieving $\geq 20 \%$ improvement in tender joint count and swollen joint count (TJC/SJC) versus baseline at two consecutive visits were discontinued from study drug.
Concomitant treatments specifically for psoriasis (eg, topicals, light therapy, retinoids) were not permitted until after week 16 psoriasis-related endpoints were evaluated. Also, from week 16, all patients who qualified for rescue therapy (ie, did not achieve $\geq 20 \%$ improvement in TJC and SJC at weeks 12 and 16 compared with baseline) were permitted to have background medication(s) initiated or changed.

\section{Patient and public involvement}

Patients and/or the public were not involved in the design, conduct, reporting or dissemination plans of this research.

\section{Assessments}

Efficacy endpoints were assessed through week 56. Online supplemental section S1 describes these assessments in detail. Importantly, changes from baseline in Bath Ankylosing Spondylitis Disease Activity Index (BASDAI) and Ankylosing Spondylitis Disease Activity Score (ASDAS) were assessed in patients with presumed psoriatic spondylitis at baseline. This determination of "psoriatic spondylitis' was presumptively made by the treating physician based on their assessment of the totality of the information available to them, which could have included previous imaging, the duration and characteristics of back pain and/or the age of onset but was not confirmed by the recognised diagnostic tests required by the classification criteria for axial spondyloarthritis. Safety reports are presented for all patients who received $\geq 1$ dose of study drug. Adverse events (AEs) were coded per the Medical Dictionary for Regulatory Activities, V.22.0; AEs and laboratory changes were graded using the National Cancer Institute Common Toxicity Criteria AE V.5.0 and V.4.03, respectively. An independent, external Cardiovascular Adjudication Committee blindly adjudicated deaths and cardiovascular events per predefined event definitions. An internal Gastrointestinal (GI) Perforation Adjudication Committee blindly adjudicated reported GI perforation events as stated in the GI perforation charter.

\section{Statistical analysis}

Efficacy analyses were conducted in the full analysis set, including all randomised patients receiving $\geq 1$ dose of study drug. For binary endpoints, treatments were compared using the Cochran-Mantel-Haenszel test, adjusting for current DMARD use (yes/no). Nonresponder imputation (NRI) was used for missing data handling. As observed (AO) data excluding missing evaluations are also shown for binary endpoints at week 56. For non-radiographic continuous endpoints, analyses were conducted using a mixed-effects model repeated measures (MMRM) model based on AO data, with fixed effects of treatment, visit, treatmentby-visit interaction, current DMARD use and the corresponding baseline value as a covariate. Missing data were handled by MMRM assuming missing at random. Analyses for radiographic endpoints were based on an 
A
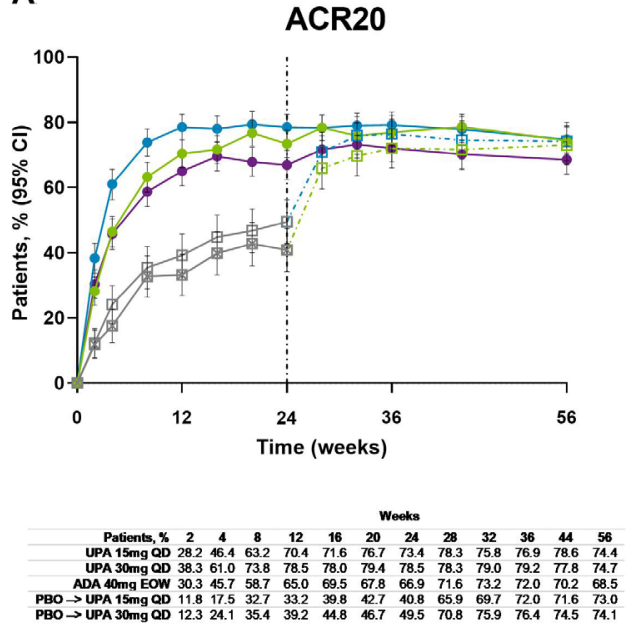

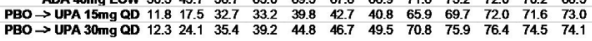

C

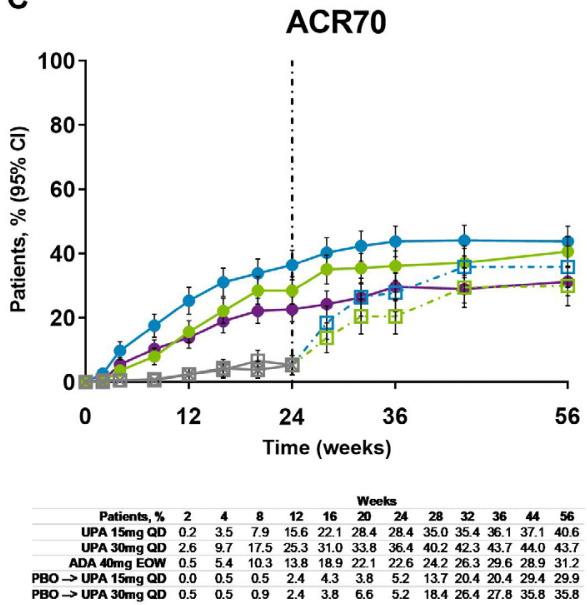

B
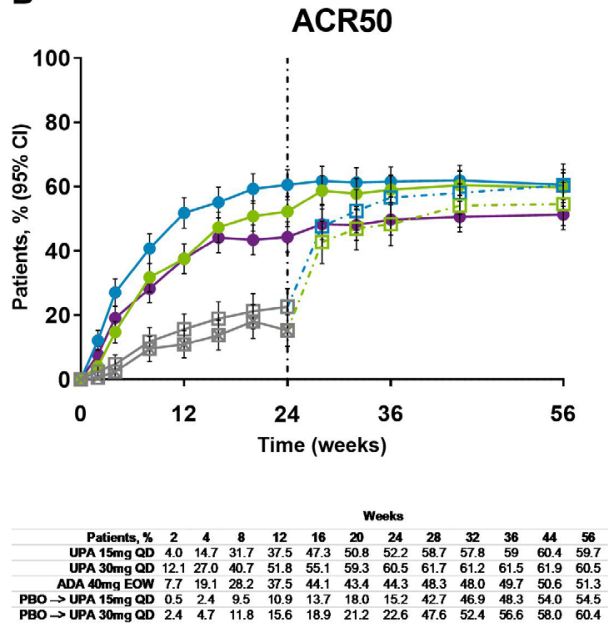

PBO $\rightarrow$ UPA 15mg OD 0.5
UPA 30mg OD

Figure 1 Proportions of patients achieving (A) ACR20, (B) ACR50 and (C) ACR70 response over 56 weeks (NRI). ACR20/50/70, $\geq 20 \% / 50 \% / 70 \%$ improvement in American College of Rheumatology response criteria; ADA, adalimumab; DMARD, disease-modifying antirheumatic drug; EOW, every other week; NRI, non-responder imputation; PBO, placebo; QD, once daily; UPA, upadacitinib. Patients originally randomised to placebo switched to either upadacitinib $15 \mathrm{mg}$ QD or upadacitinib $30 \mathrm{mg}$ QD (1:1) at week 24 and their data up to week 24 are under placebo exposure. 95\% Cls for response rate were calculated based on normal approximation to the binominal distribution. Nominal $p$ value was constructed using Cochran-Mantel-Haenszel test adjusted for the main stratification factor of current DMARD use (yes/no).

analysis of covariance model including treatment and current DMARD use as fixed factors and baseline value as a covariate, with linear extrapolation used as the primary approach for missing data handling. Patients originally randomised to placebo were switched to upadacitinib $15 \mathrm{mg}$ or $30 \mathrm{mg}$ at week 24 and summarised by 'placebo to upadacitinib $15 \mathrm{mg}$ or $30 \mathrm{mg}$ '. Treatment comparisons between each upadacitinib dose versus adalimumab were conducted for the originally randomised upadacitinib groups and adalimumab for all non-radiographic endpoints; nominal $\mathrm{p}$ values are presented for weeks 12, 24 and 56.

For safety analyses, the upadacitinib $15 \mathrm{mg}$ and $30 \mathrm{mg}$ groups included patients who were originally randomised to placebo and switched to upadacitinib at week 24 . Treatment-emergent AEs (TEAEs) were summarised for events occurring while exposed to upadacitinib or adalimumab until the last subject reached week 56 ; exposureadjusted event rates per 100 patient years (PY; E/100
PY) were summarised as events based on the treatment received at the time of each $\mathrm{AE}$, during the time between the first and last dose of upadacitinib or adalimumab, and up to 30 or 70 days after, respectively, if the patient discontinued prematurely from the study; multiple events occurring in the same patient were included in the numerator and 95\% CIs were calculated. Exposureadjusted incidence rates per $100 \mathrm{PY}$ were summarised as the number of patients with $\geq 1$ event/100 PY ( $\mathrm{n} / 100 \mathrm{PY})$, with exposure calculated up to onset of the first event; multiple events occurring in the same patient were not included in the numerator and $95 \%$ CIs were calculated.

\section{RESULTS}

\section{Patients}

Of 1705 patients randomised, 1419 (83.2\%) completed 56 weeks of treatment (online supplemental figure S1). The most common reasons for study discontinuation 
A

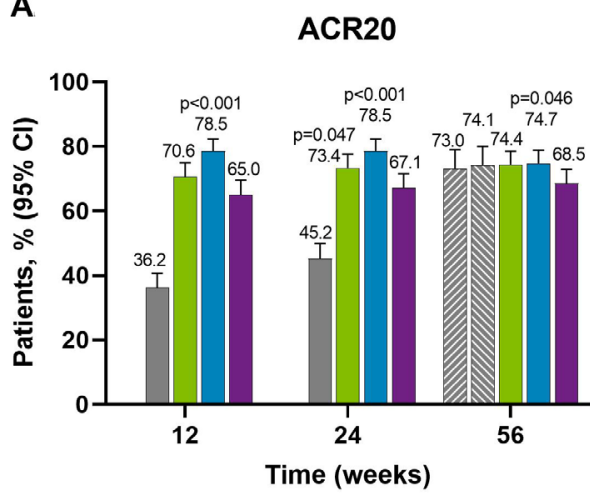

C

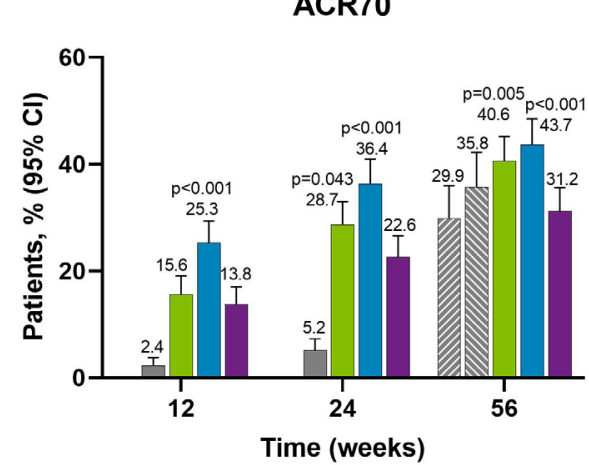

B

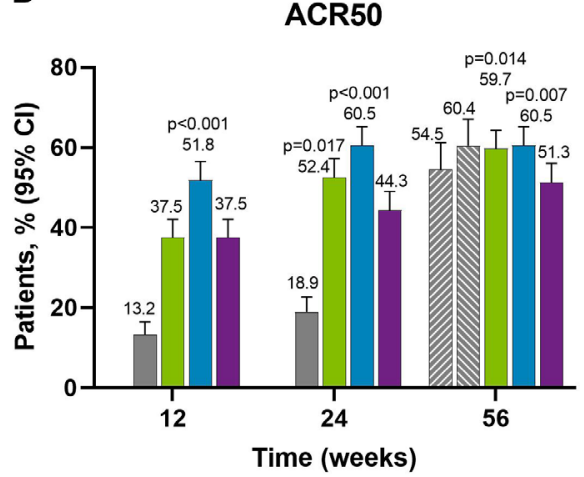

Figure 2 Proportions of patients achieving (A) ACR20, (B) ACR50 and (C) ACR70 response at weeks 12, 24 and 56 (NRI). Nominal p values are for upadacitinib versus adalimumab. ACR20/50/70, $\geq 20 \% / 50 \% / 70 \%$ improvement in American College of Rheumatology response criteria; ADA, adalimumab; DMARD, disease-modifying antirheumatic drug; EOW, every other week; NRI, non-responder imputation; PBO, placebo; QD, once daily; UPA, upadacitinib. For the week 56 data, patients originally randomised to placebo switched to either upadacitinib $15 \mathrm{mg}$ QD or upadacitinib $30 \mathrm{mg}$ QD $(1: 1)$ at week 24 and their data up to week 24 are under placebo exposure. 95\% Cls for response rate were calculated based on normal approximation to the binominal distribution. Nominal $p$ value was constructed using Cochran-Mantel-Haenszel test adjusted for the main stratification factor of current DMARD use (yes/no).

across all treatment groups were withdrawal by patient and lack of efficacy. As reported previously, baseline characteristics were balanced across groups (online supplemental table S1). ${ }^{16}$

\section{Efficacy}

Consistent with previously reported week 24 results, ${ }^{16}$ upadacitinib continued to demonstrate efficacy at week 56 across key domains of PsA including musculoskeletal and skin outcomes and patient-reported outcomes (PROs).

\section{Musculoskeletal outcomes}

Across all treatment groups, the proportions of patients achieving ACR20/50/70 response were maintained from week 24 through week 56 , with greater proportions of patients originally randomised to upadacitinib $15 \mathrm{mg}$ and $30 \mathrm{mg}$ achieving ACR20/50/70 compared with adalimumab at week 56 (NRI analysis; nominal $\mathrm{p} \leq 0.05$ for upadacitinib $15 \mathrm{mg}$ versus adalimumab for ACR50/70; nominal $\mathrm{p} \leq 0.05$ for upadacitinib $30 \mathrm{mg}$ versus adalimumab for ACR20/50/70) (figures 1 and 2). Improvements were observed for all ACR components (table 1). At week 56, patients originally randomised to placebo showed a similar ACR20/50/70 response following switch to upadacitinib at week 24. Individual patient responses for ACR20/50/70 over time, including the time course of achievement and sustainability of these responses are presented in online supplemental figure S1.

The proportion of patients achieving Psoriatic Arthritis Response Criteria (PsARC) was also maintained from week 24 through week 56 in all treatment groups; more patients achieved PsARC response with upadacitinib $30 \mathrm{mg}$ versus adalimumab at week 56 (nominal $\mathrm{p} \leq 0.05$; online supplemental figure S3).

At week 56, similar proportions of patients originally randomised to upadacitinib $15 \mathrm{mg}$, upadacitinib $30 \mathrm{mg}$ or adalimumab achieved resolution of enthesitis or dactylitis; these proportions were maintained or increased compared with week 24 (online supplemental figure S4).

At week 56, patients showed improvement in Disease Activity in Psoriatic Arthritis (table 1), and patients with evidence of psoriatic spondylitis at baseline showed improvements in ASDAS and BASDAI. Comparable results at week 56 were observed in patients who switched 


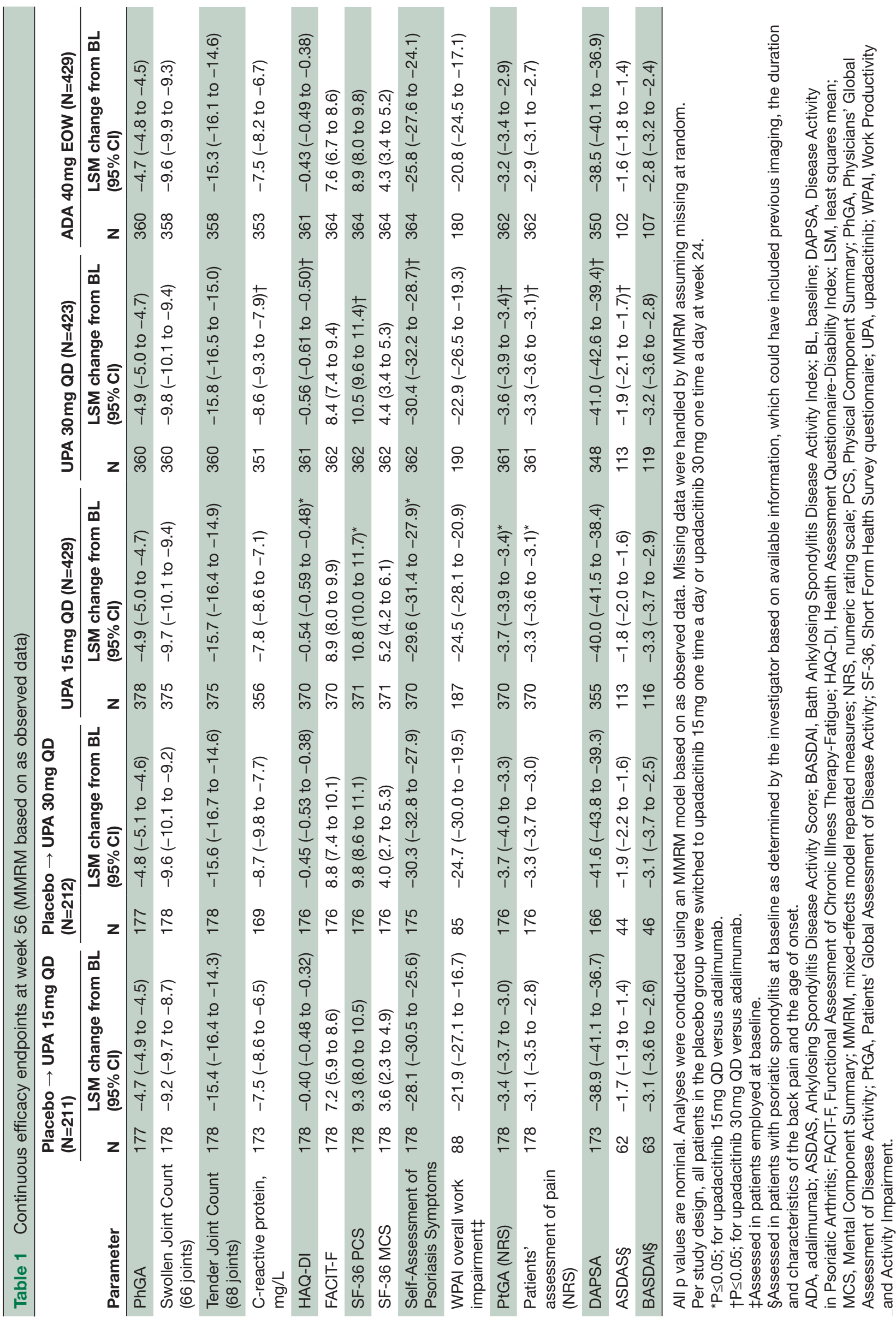


A Linear Extrapolation
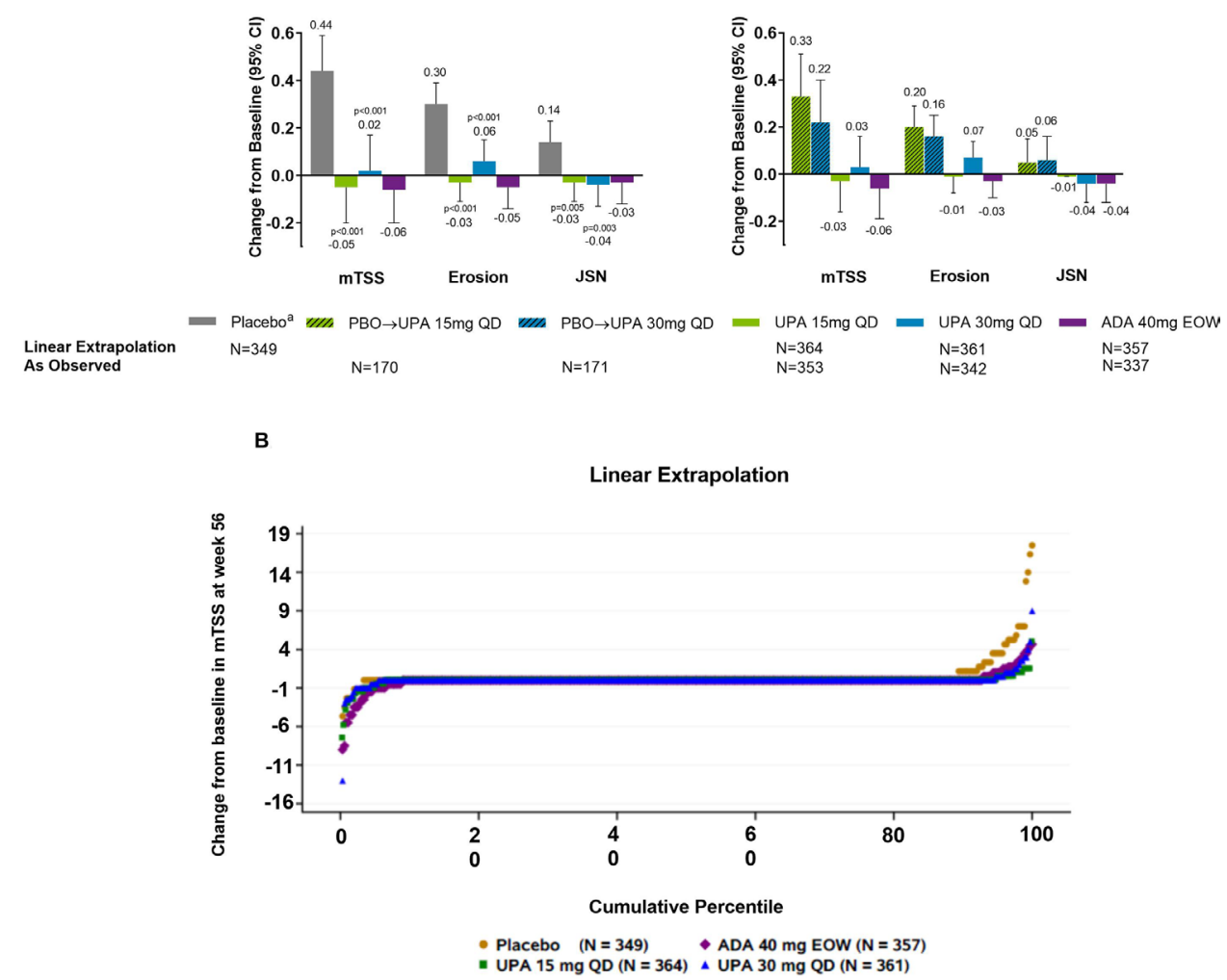

Figure 3 (A) Change from baseline at week 56 in radiographic endpoints. (B) Probability plot of change from baseline in mTSS at week 56 (linear extrapolation). Nominal p values are for upadacitinib versus placebo. ADA, adalimumab; DMARD, diseasemodifying antirheumatic drug; EOW, every other week; JSN, joint space narrowing score; mTSS, modified total Sharp/van der Heijde Score; PBO, placebo; QD, once daily; UPA, upadacitinib. Patients originally randomised to placebo switched to either upadacitinib $15 \mathrm{mg}$ QD or upadacitinib $30 \mathrm{mg}$ QD (1:1) at week 24 and their data up to week 24 are under placebo exposure. Least square mean and $95 \% \mathrm{Cls}$ and nominal $\mathrm{p}$ values are based on an analysis of covariance model including treatment and the stratification factor current DMARD use (yes/no) as fixed factors and baseline value as covariate.

from placebo to upadacitinib compared with patients originally randomised to upadacitinib (table 1 ).

Mean changes from baseline in radiographic endpoints were comparable with upadacitinib $15 \mathrm{mg}$, upadacitinib $30 \mathrm{mg}$ and adalimumab based on linear extrapolation at week 56 (figure 3). As linear extrapolation assumes results would follow the same trend regardless of switch or discontinuation, $\mathrm{AO}$ analyses were also conducted with similar results observed (figure 3). At week 56, the rates of non-progression were greater with upadacitinib $15 \mathrm{mg}$ and $30 \mathrm{mg}$ versus those initially randomised to placebo based on linear extrapolation analysis (nominal $\mathrm{p} \leq 0.05$; online supplemental figure S5).

The proportions of overall patients achieving MDA were maintained from week 24 through week 56 in patients originally randomised to upadacitinib $(36.6 \%$ and $45.4 \%$ for upadacitinib $15 \mathrm{mg}$ and $30 \mathrm{mg}$, respectively, at week 24 , and $44.8 \%$ and $47.3 \%$ at week 56 ) or adalimumab (33.3\%-39.6\%); more upadacitinib 30 mg-treated patients achieved MDA versus adalimumab at week 56 (nominal $\mathrm{p} \leq 0.05$; figure 4 ). An increase in patients achieving MDA was observed for those originally randomised to placebo and switched to upadacitinib. Individual patient response for MDA over time showed that most patients who achieved MDA maintained the response through week 56 (online supplemental figure S6).

\section{Skin outcomes}

Improvements in skin outcomes were maintained over time in Psoriasis Area and Severity Index (PASI75/90/100) and Static Investigator Global Assessment of Psoriasis of 0 or 1 (sIGA 0/1) response rates (figure 5), and change from baseline in Self-Assessment of Psoriasis Symptoms (table 1). In patients randomised to placebo, the proportion of patients achieving PASI75/90/100 and sIGA 0/1 increased following switch to upadacitinib, and responses were similar to the upadacitinib groups at week 56 . Individual patient PASI75/90 responses over time for all treatment groups, including the time course of achievement and sustainability of these responses, are presented in online supplemental figure S7 .

\section{Patient-reported outcomes}

Improvements were maintained from week 24 through week 56 in Health Assessment Questionnaire-Disability Index (HAQ-DI), Functional Assessment of Chronic Illness Therapy-Fatigue, Short Form Health Survey 

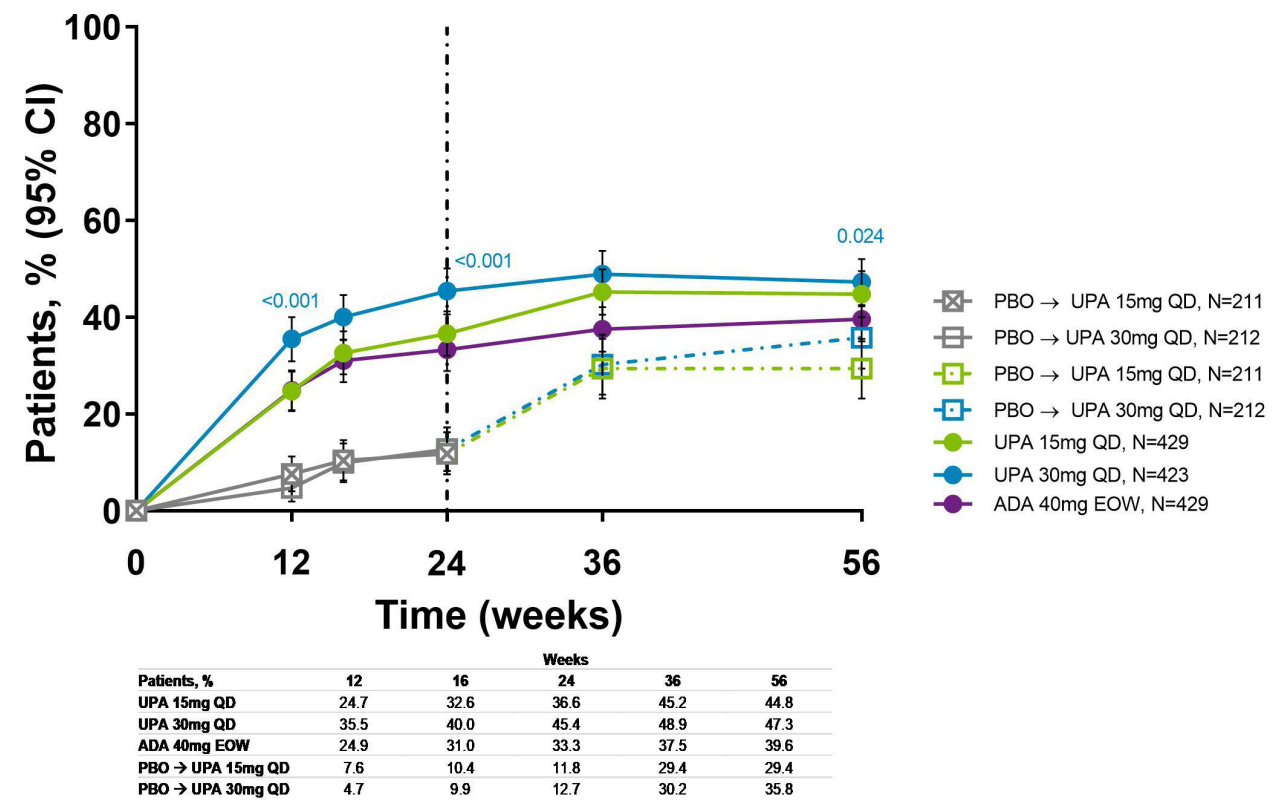

Figure 4 Proportion of patients achieving MDA over 56 weeks (NRI). Nominal $p$ values are for upadacitinib versus adalimumab. ADA, adalimumab; DMARD, disease-modifying antirheumatic drug; EOW, every other week; MDA, minimal disease activity; NRI, non-responder imputation; PBO, placebo; QD, once daily; UPA, upadacitinib. Patients originally randomised to placebo switched to either upadacitinib $15 \mathrm{mg}$ QD or upadacitinib $30 \mathrm{mg}$ QDy (1:1) at week 24 and their data up to week 24 are under placebo exposure. NRI with additional rescue handling was used, where patients rescued at week 16 are imputed as non-responders. $95 \% \mathrm{Cls}$ for response rate were calculated based on normal approximation to the binominal distribution. Nominal p value was constructed using Cochran-Mantel-Haenszel test adjusted for the main stratification factor of current DMARD use (yes/no).

questionnaire (SF-36) Physical Component Summary (PCS) and Mental Component Summary, patients' assessment of pain, Patients' Global Assessment of Disease Activity and Work Productivity and Activity Impairment. Similar proportions of patients across all treatment groups achieved $\geq 30 \%$ reduction in baseline pain and $\geq 50 \%$ reduction in baseline pain at week 56 (online supplemental figure S8). Furthermore, greater improvement was observed in the upadacitinib $15 \mathrm{mg}$ and $30 \mathrm{mg}$ groups compared with the adalimumab group for change from baseline in HAQ-DI and SF-36 PCS (nominal $\mathrm{p} \leq 0.05$ for all comparisons; table 1 ). At week 56, improvements in PROs for patients randomised to placebo generally reached similar levels to those observed in patients randomised to upadacitinib.

The proportion of patients achieving HAQ-DI minimally clinically important difference (MCID) (improvement in HAQ-DI total score of $\geq 0.35$ from baseline) or a normative HAQ-DI ${ }^{17}$ (HAQ-DI score $\leq 0.25$ ) at week 24 continued to increase or was maintained through week 56. Compared with the adalimumab group, the proportion of patients who achieved an MCID in HAQ-DI was greater in the upadacitinib $15 \mathrm{mg}$ and $30 \mathrm{mg}$ groups at week 56 (nominal $\mathrm{p}<0.05$ ) (online supplemental figures S9 and S10).

Numerically greater improvements in musculoskeletal, skin and PROs were generally observed at week 56 using AO analysis compared with NRI analysis (online supplemental table S2).

\section{Safety}

Through week 56, rates of TEAEs were higher with upadacitinib $30 \mathrm{mg}$ versus upadacitinib $15 \mathrm{mg}$ and adalimumab (333.9 vs 281.1 and $265.9 \mathrm{E} / 100 \mathrm{PY}$, respectively). Rates of serious AEs were also higher with upadacitinib $30 \mathrm{mg}$ versus upadacitinib $15 \mathrm{mg}$ and adalimumab (12.3 vs 9.1 and $9.3 \mathrm{E} / 100 \mathrm{PY}$, respectively). The most commonly reported AEs were upper respiratory tract infection and blood creatine phosphokinase (CPK) elevations (online supplemental table S3). Two deaths were reported with upadacitinib $15 \mathrm{mg}$ (one from metastatic lung cancer and one from lower respiratory tract infection), two with upadacitinib $30 \mathrm{mg}$ (one from coronavirus infection and one from interstitial lung disease) and one with adalimumab (due to a traffic accident). One death was reported in the placebo group during the 24-week placebo-controlled period in a patient who experienced an unspecified emergency while driving. These deaths are described in detail in online supplemental figure $\mathrm{S} 2$.

Up to week 56, the rate of serious infections was 2.9, 4.7 and $1.3 \mathrm{E} / 100 \mathrm{PY}$ with upadacitinib $15 \mathrm{mg}$, upadacitinib $30 \mathrm{mg}$ and adalimumab, respectively (figure 6). Treatment-emergent opportunistic infections included one event each of candida urethritis, bronchopulmonary aspergillosis and oral fungal infection with upadacitinib $15 \mathrm{mg}$; one event each of cytomegalovirus infection, oropharyngeal candidiasis and 

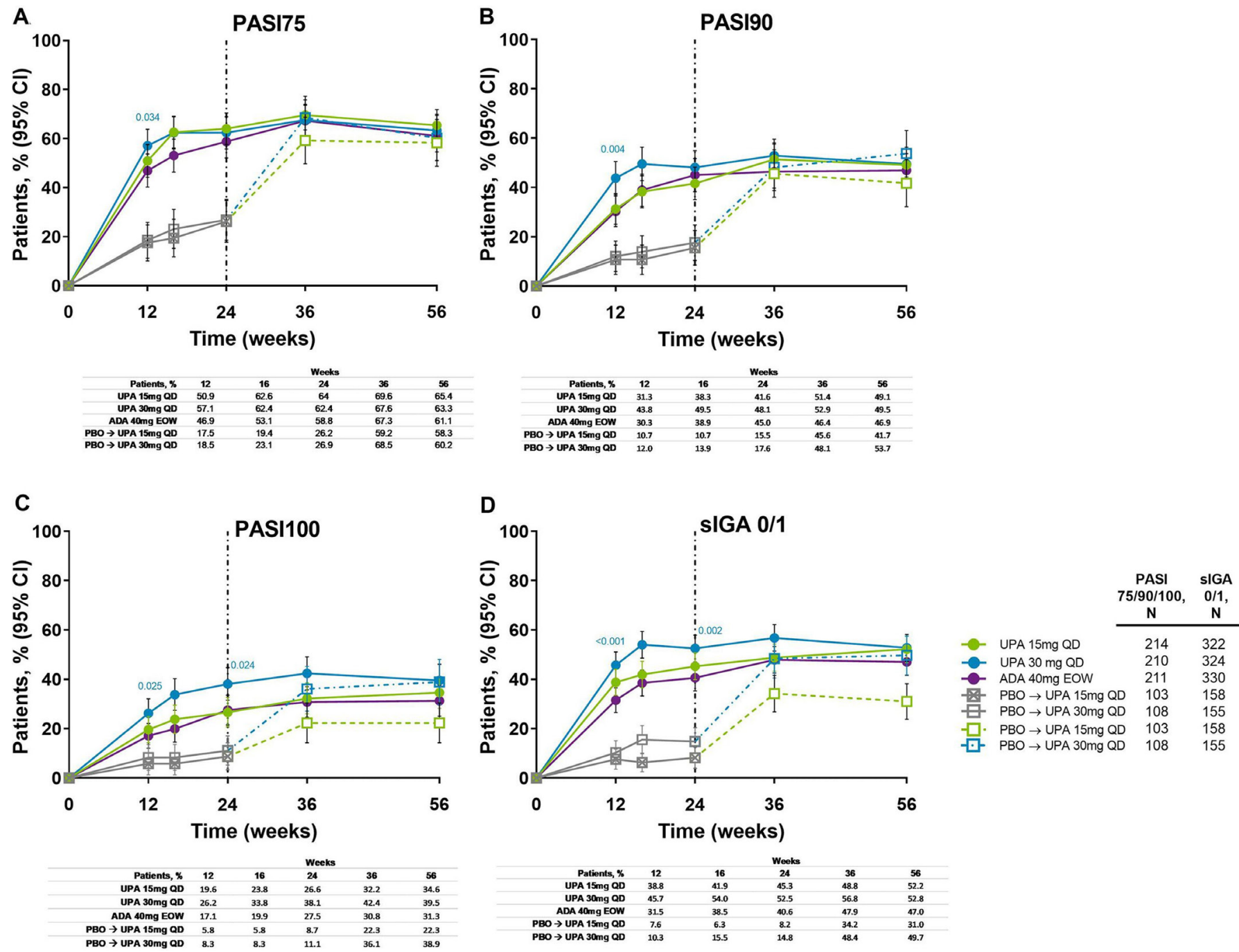

Figure 5 Proportion of patients achieving (A) PASI75, (B) PASI90, (C) PASI100 and (D) sIGA 0/1 response over 56 weeks (NRI). Nominal $p$ values are for upadacitinib versus adalimumab. ADA, adalimumab; DMARD, disease-modifying antirheumatic drug; EOW, every other week; NRI, non-responder imputation; PASI75/90/100, $\geq 75 \% / 90 \% / 100 \%$ improvement in Psoriasis Area Severity Index; PBO, placebo; QD, once daily; sIGA, Static Investigator Global Assessment of Psoriasis; UPA, upadacitinib. After week 16 assessments have been performed, patients may use concomitant treatments specifically for psoriasis per investigator judgement. Patients originally randomised to placebo switched to either upadacitinib $15 \mathrm{mg}$ QD or upadacitinib $30 \mathrm{mg}$ QD (1:1) at week 24 and their data up to week 24 are under placebo exposure. 95\% Cls for response rate were calculated based on normal approximation to the binominal distribution. Nominal $p$ value was constructed using CochranMantel-Haenszel test adjusted for the main stratification factor of current DMARD use (yes/no).

pneumocystis jirovecii pneumonia; and four events of oral fungal infection with upadacitinib $30 \mathrm{mg}$. No cases of active tuberculosis were reported. The rate of herpes zoster (HZ) was 3.9, 6.4 and $0.5 \mathrm{E} / 100 \mathrm{PY}$ with upadacitinib $15 \mathrm{mg}$, upadacitinib $30 \mathrm{mg}$ and adalimumab, respectively; most events were mild/moderate in severity, limited to one to two dermatomes, and did not lead to study drug discontinuation. Most patients experiencing an $\mathrm{HZ}$ event had not had a prior $\mathrm{HZ}$ vaccination.

Malignancy event rates were similar with upadacitinib $15 \mathrm{mg}$, upadacitinib $30 \mathrm{mg}$ and adalimumab, and no notable pattern or types of malignancies were observed. Most events of non-melanoma skin cancer were mild/moderate in severity, non-serious and did not lead to study drug discontinuation. Two basal cell carcinoma events led to study drug discontinuation (one in each of the upadacitinib groups). Two nonfatal strokes and one non-fatal myocardial infarction (MI) were reported in each of the upadacitinib $15 \mathrm{mg}$ and adalimumab groups, and two non-fatal MIs were reported with upadacitinib $30 \mathrm{mg}$. These events are described in detail in online supplemental section S3. Ten venous thromboembolic events were reported in nine patients. One event of deep vein thrombosis (DVT) was reported in each of the upadacitinib groups and two with adalimumab; one event of pulmonary embolism (PE) was reported with upadacitinib $15 \mathrm{mg}$ and three events with upadacitinib $30 \mathrm{mg}$. One patient in the upadacitinib $15 \mathrm{mg}$ group had concurrent DVT 

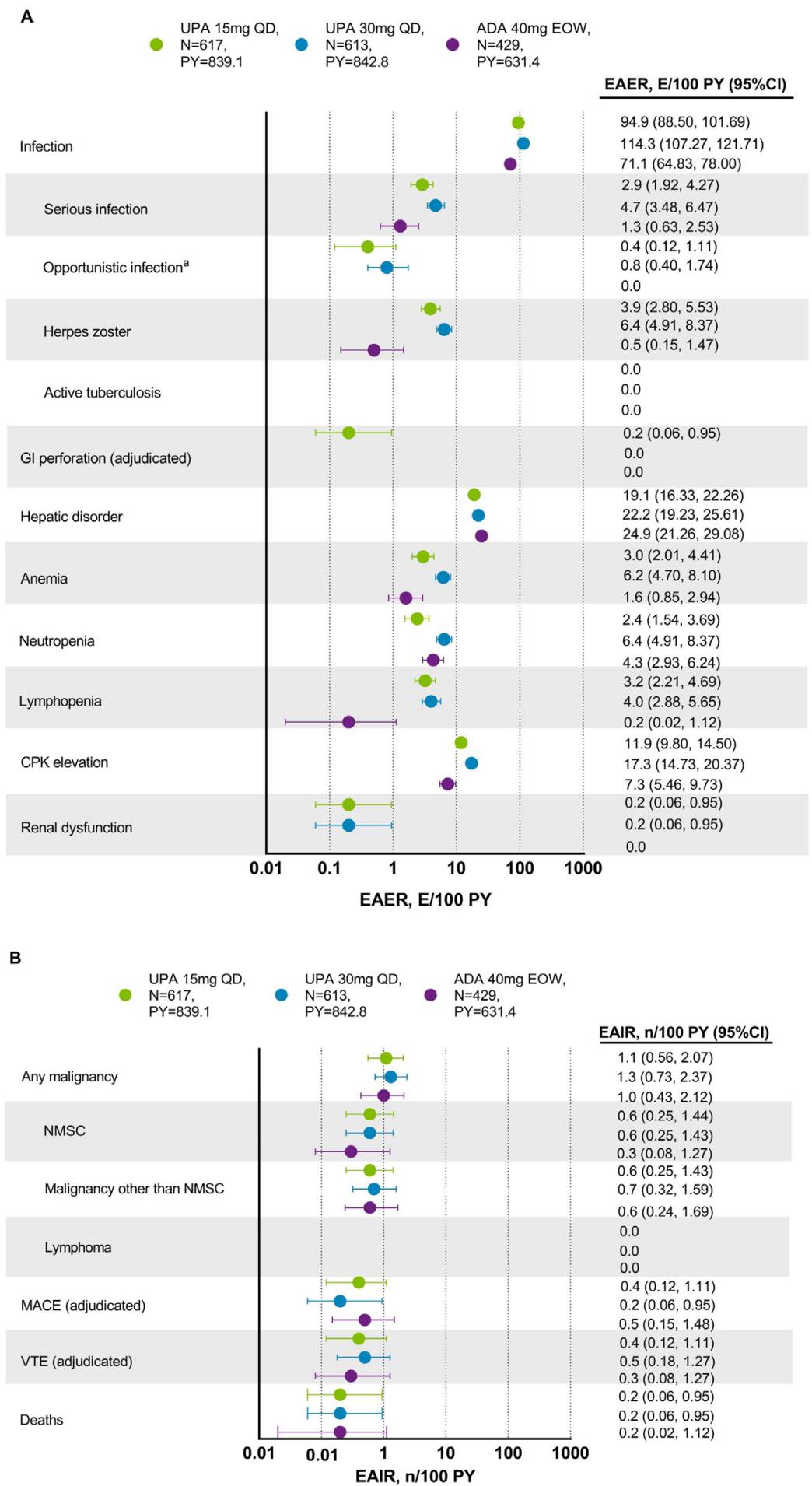

Figure 6 (A) Exposure-adjusted event and (B) incidence rates of treatment-emergent AEs through week 56. ${ }^{a}$ Excluding tuberculosis and herpes zoster. ADA, adalimumab; AE, adverse event; CPK, creatine phosphokinase; EAER, exposure-adjusted event rate; EAIR, exposure-adjusted incidence rate; EOW, every other week; Gl, gastrointestinal; MACE, major adverse cardiovascular events (defined as non-fatal myocardial infarction, non-fatal stroke and cardiovascular death); NMSC, nonmelanoma skin cancer; PY, patient years; QD, once daily; UPA, upadacitinib; VTE, venous thromboembolism (defined as deep vein thrombosis and pulmonary embolism). There were 11 malignancies reported in each of the upadacitinib $15 \mathrm{mg}$ (4 basal cell carcinomas, 2 squamous cell carcinoma of skin and 1 event each of endometrial adenocarcinoma, lung adenocarcinoma, lung cancer metastatic, malignant melanoma and neuroendocrine carcinoma) and upadacitinib $30 \mathrm{mg}$ groups (2 basal cell carcinomas, 2 squamous cell carcinoma of skin and 1 event each of adenocarcinoma of colon, Bowen's disease, breast cancer, clear cell renal cell carcinoma, invasive breast carcinoma, lung adenocarcinoma and plasma cell myeloma), and six malignancies reported with adalimumab (two basal cell carcinomas and one event each of colon cancer metastatic, ovarian cancer, pancreatic carcinoma metastatic and uterine cancer). 
and PE. These events are described in detail in online supplemental section $\mathrm{S} 4$.

Grade 3 decreases in haemoglobin, platelets, lymphocytes and neutrophils occurred in $\leq 4 \%$ of patients in each group; most events of lymphopenia were isolated, resolved without interruption in therapy and were not associated with bacterial, opportunistic, fungal or viral infections (online supplemental table S4). Grade 4 decreases in haemoglobin, platelets, lymphocytes and neutrophils were reported in $\leq 1 \%$ of patients in each group. However, post database lock, it was determined that all grade 4 decreases were captured due to data entry errors by the site and were not considered potentially clinically significant. AEs of anaemia and lymphopenia were more common with either dose of upadacitinib versus adalimumab and with upadacitinib $30 \mathrm{mg}$ versus upadacitinib $15 \mathrm{mg}$. AEs of neutropenia were more common with upadacitinib $30 \mathrm{mg}$ and adalimumab versus upadacitinib $15 \mathrm{mg}$.

The rate of hepatic disorder AEs was 19.1, 22.2 and $24.9 \mathrm{E} / 100$ PY with upadacitinib $15 \mathrm{mg}$, upadacitinib $30 \mathrm{mg}$ and adalimumab, respectively. Most alanine aminotransferase (ALT) or aspartate aminotransferase (AST) increases were mild/moderate (grade 2 or less) and transient. Grade 3 increases in ALT or AST were observed in $<2.1 \%$ of patients across all groups. No Hy's law cases were reported. Grade 3 or 4 CPK increases were more common with upadacitinib and were reported in $<5.7 \%$ of patients; CPK increases were generally asymptomatic (one patient on upadacitinib $30 \mathrm{mg}$ had CPK elevation $>10 \times$ upper limit of normal and experienced dermatomyositis approximately 34 days after discontinuing treatment due to a prior event of bronchitis), and no patients experienced rhabdomyolysis.

\section{DISCUSSION}

SELECT-PsA 1 is a large study in patients with PsA who have had IR or intolerance to $\geq 1$ non-biological DMARD and includes adalimumab as an active comparator; as such, this study offers the opportunity to understand the maintained efficacy and safety of upadacitinib in the context of current standard of care treatment for PsA. In this 56-week analysis, upadacitinib continued to demonstrate improvements in most clinically relevant manifestations of PsA including musculoskeletal and skin symptoms, physical function, QoL and other PROs, as well as inhibiting radiographic progression. In addition, the proportion of patients achieving MDA at week 24 continued to increase through week 56 . Notably, upadacitinib continued to show results that were comparable with those of adalimumab at week 56, with results for some endpoints being significantly greater based on nominal $p$ values. At week 56, improvements in patients who switched from placebo to upadacitinib were generally comparable, except for resolution of enthesitis and dactylitis, to those originally randomised to upadacitinib. Although not unexpected, it is encouraging to see that patients originally treated with placebo rapidly improved between weeks 24 and 56 after switching to upadacitinib, and reached similar levels of improvement as those originally randomised to upadacitinib.

Safety over 56 weeks remained consistent with observations through week 24 and the upadacitinib RA trials. ${ }^{11-1618}$ Event rates of serious and opportunistic infections and $\mathrm{HZ}$ were greater with upadacitinib versus adalimumab. Treatment-emergent malignancies, major adverse cardiovascular events and venous thromboembolism appeared comparable across treatment groups.

A major limitation of the axial data presented from this trial includes the lack of axial imaging to assess for psoriatic spondylitis; the diagnosis was made on presumptive criteria that could have included patients without true spondylitis. As magnetic resonance images and radiographs of the sacroiliac joints and axial skeleton were not required to confirm evidence of inflammation in the spine nor radiographic changes in the spine or sacroiliac joints, the presence of 'psoriatic spondylitis' was based on the totality of the information available to the treating physician, which may have been subjective and may not have included imaging. This poses a potential major limitation to the interpretation of the results pertaining to axial symptoms. Another limitation was that this 56-week study was not powered or designed to include a prespecified statistical comparison for efficacy between the upadacitinib groups and adalimumab through week 56 .

In summary, efficacy responses were maintained with upadacitinib $15 \mathrm{mg}$ and $30 \mathrm{mg}$ treatment over 56 weeks and were generally numerically higher than with adalimumab. The significant inhibition of radiographic progression at week 24 was maintained at week 56 and was similar in the upadacitinib and adalimumab groups. Additionally, at week 56, improvements in efficacy were observed in patients who switched from placebo to upadacitinib. No new safety findings were observed with longer term exposure to upadacitinib.

\section{Author affiliations}

${ }^{1}$ Institute of Infection, Immunity \& Inflammation, University of Glasgow, Glasgow, UK ${ }^{2}$ Department of Immunology, AbbVie Inc, North Chicago, Illinois, USA

${ }^{3}$ School of Medicine at Metrohealth Medical Center, Case Western Reserve University, Cleveland, Ohio, USA

${ }^{4}$ Department of Rheumatology, Brigham and Women's Hospital and Harvard Medical School, Boston, Massachusetts, USA

${ }^{5}$ Department of Nephrology and Rheumatology, Kyorin University School of Medicine, Tokyo, Japan

${ }^{6}$ Facultad de Medicina, Universidad Autónoma de Chihuahua, Chihuahua, Mexico ${ }^{7}$ Department of Medicine, McMaster University, Hamilton, Ontario, Canada

${ }^{8}$ Department of Rheumatology, The Waterside Clinic, Barrie, Ontario, Canada ${ }^{9}$ Department of Immunology, AbbVie Deutschland GmbH \& Co KG, Wiesbaden, Hesse, Germany

${ }^{10}$ Department of Rheumatology, Goethe University \& Fraunhofer IME-TMP and CIMD, Frankfurt, Germany 
Correction notice This article has been corrected since it was first published. The author name 'Frank Behrens' was incorrectly spelt as 'Franck Behrens'.

Acknowledgements AbbVie and the authors thank the patients, trial sites and investigators who participated in this clinical trial. AbbVie was the trial sponsor, contributed to trial design, data collection, analysis and interpretation, and to writing, review and approval of final version. No honoraria or payments were made for authorship. Medical writing support was provided by Ramona Vladea, PhD of AbbVie and Laura Chalmers, PhD of 2 the Nth (Cheshire, UK), and was funded by AbbVie.

Contributors ALP, JL and KK participated in the design of the study. LC, YD, IBM, MM, JFM, MK, CP-T and DH participated in the acquisition of data. ALP, KK, LC, YD, $\mathrm{PZ}, \mathrm{JL}, \mathrm{RL}, \mathrm{IBM}, \mathrm{MM}, \mathrm{JFM}, \mathrm{MK}, \mathrm{CP}-\mathrm{T}$ and $\mathrm{DH}$ participated in the interpretation of data. LC and YD participated in the analysis of data. ALP, KK and IBM contributed to the drafting of the manuscript. All authors contributed to the critical revision of the manuscript for important intellectual content

Funding AbbVie funded this study and participated in the study design, research, analysis, data collection, interpretation of data, review and approval of the publication. All authors had access to relevant data and participated in the drafting, review and approval of this publication. No honoraria or payments were made for authorship.

Competing interests IBM: research grants and honoraria from AbbVie, BristolMyers Squibb, Celgene, Eli Lilly, Gilead, Janssen, Novartis, Pfizer, Sanofi Regeneron and UCB Pharma. MM: research grants from AbbVie, Amgen and UCB Pharma; consulting fees from Eli Lilly, Janssen, Novartis, Pfizer and UCB Pharma. JFM: consultant and/or investigator for AbbVie, Arena, Avotres, Biogen, Bristol-Myers Squibb, Celgene, Dermavant, Eli Lilly, EMD Sorono, Janssen, Leo Pharma, Merck, Novartis, Pfizer, Regeneron, Sanofi, Sun Pharma and UCB Pharma. MK: consulting fees and/or honoraria from AbbVie, AmgenAstellas BioPharma, Asahi-Kasei Pharma, Astellas, Ayumi Pharma, Bristol-Myers Squibb, Chugai, DaiichiSankyo, Eisai, Eli Lilly, Gilead, Janssen, Kyowa Kirin, Novartis, Ono Pharma, Pfizer, Tanabe-Mitsubishi, Teijin Pharma and UCB Pharma. CP-T: research grants and honoraria form AbbVie, AstraZeneca, Eli Lilly, Gilead, Janssen, Pfizer, Roche, R-Pharm, Sanofi Regeneron and UCB Pharma. DH: advisory board/speaker bureau or similar committee for AbbVie, Amgen, AstraZeneca, Bristol-Myers Squibb, GlaxoSmithKline, Janssen, Novartis, Pfizer, Roche, Sanofi Genzyme and Takeda; funded grant or clinical trials: AbbVie, Adiga Life-Sciences, Amgen, Bristol-Myers Squibb, Can-Fite Biopharma, Celgene, Eli Lilly, Gilead, GlaxoSmithKline, Janssen, Novartis, Pfizer, Regeneron, Sanofi-Genzyme and UCB Pharma; honoraria or other fees from AbbVie, Amgen, AstraZeneca, Bristol-Myers Squibb, Eli Lilly, GlaxoSmithKline, Janssen, Merck, Novartis, Pfizer, Roche, Sanofi Genzyme, Takeda and UCB Pharma; not a part of/has not received payment from a commercial organisation (gifts or 'in kind' compensation); does not hold a patent for a product referred to in the CME/CPD program, or marketed by a commercial organisation; does not hold investments in a pharmaceutical organisation, medical devices company or communications firm. FB: research grants from Celgene, Chugai, Janssen, Pfizer and Roche; consultancies/speaker fees from AbbVie, Bristol-Myers Squibb, Boehringer, Celgene, Chugai, Eli Lilly, Genzyme, Janssen, MSD, Novartis, Pfizer, Roche, Sanofi and UCB Pharma. KK, ALP, YD, LC, PZ, RL, JL: AbbVie employees and may own AbbVie stock or options.

Patient consent for publication Not applicable.

Ethics approval The study was conducted according to the International Conference on Harmonization guidelines, applicable regulations and guidelines governing clinical trial conduct, and the Declaration of Helsinki. The trial protocol was approved by independent ethics committees and institutional review boards, and all patients provided written informed consent.

Provenance and peer review Not commissioned; externally peer reviewed.

Data availability statement Data are available upon reasonable request. These clinical trial data can be requested by any qualified researchers who engage in rigorous, independent scientific research and will be provided following review and approval of a research proposal and statistical analysis plan and execution of a data-sharing agreement. Data requests can be submitted at any time and the data will be accessible for 12 months, with possible extensions considered. For more information on the process or to submit a request, visit the following website: https://www.abbvie.com/our-science/clinical-trials/clinical-trials-data-andinformation-sharing/data-and-information-sharing-with-qualified-researchers.html.

Open access This is an open access article distributed in accordance with the Creative Commons Attribution Non Commercial (CC BY-NC 4.0) license, which permits others to distribute, remix, adapt, build upon this work non-commercially, and license their derivative works on different terms, provided the original work is properly cited, appropriate credit is given, any changes made indicated, and the use is non-commercial. See: http://creativecommons.org/licenses/by-nc/4.0/.
ORCID iDs

lain B Mclnnes http://orcid.org/0000-0003-4449-8501

Mitsumasa Kishimoto http://orcid.org/0000-0002-4007-1589

\section{REFERENCES}

1 Coates LC, Kavanaugh A, Mease PJ, et al. Group for research and assessment of psoriasis and psoriatic arthritis 2015 treatment recommendations for psoriatic arthritis. Arthritis Rheumatol 2016;68:1060-71.

2 Gossec L, Baraliakos X, Kerschbaumer A, et al. EULAR recommendations for the management of psoriatic arthritis with pharmacological therapies: 2019 update. Ann Rheum Dis 2020;79:700-12.

3 Coates LC, Helliwell PS. Treat to target in psoriatic arthritis-evidence, target, research agenda. Curr Rheumatol Rep 2015;17:517.

4 Coates LC, Moverley AR, McParland L, et al. Effect of tight control of inflammation in early psoriatic arthritis (TICOPA): a UK multicentre, open-label, randomised controlled trial. Lancet 2015;386:2489-98.

5 Deodhar A, Gottlieb AB, Boehncke W-H, et al. Efficacy and safety of guselkumab in patients with active psoriatic arthritis: a randomised, double-blind, placebo-controlled, phase 2 study. Lancet 2018;391:2213-24.

6 Mclnnes IB, Kavanaugh A, Gottlieb AB, et al. Efficacy and safety of ustekinumab in patients with active psoriatic arthritis: 1 year results of the phase 3 , multicentre, double-blind, placebo-controlled PSUMMIT 1 trial. Lancet 2013;382:780-9.

7 Mease P, Coates LC, Helliwell PS, et al. Efficacy and safety of filgotinib, a selective Janus kinase 1 inhibitor, in patients with active psoriatic arthritis (EQUATOR): results from a randomised, placebocontrolled, phase 2 trial. Lancet 2018;392:2367-77.

8 Mease P, Hall S, FitzGerald O, et al. Tofacitinib or adalimumab versus placebo for psoriatic arthritis. N Engl J Med 2017;377:1537-50.

9 Mease P, van der Heijde D, Landewé R, et al. Secukinumab improves active psoriatic arthritis symptoms and inhibits radiographic progression: primary results from the randomised, double-blind, phase III future 5 study. Ann Rheum Dis 2018;77:890-7.

10 Mease PJ, van der Heijde D, Ritchlin CT, et al. Ixekizumab, an interleukin-17A specific monoclonal antibody, for the treatment of biologic-naive patients with active psoriatic arthritis: results from the 24-week randomised, double-blind, placebo-controlled and active (adalimumab)-controlled period of the phase III trial SPIRIT-P1. Ann Rheum Dis 2017;76:79-87.

11 Burmester GR, Kremer JM, Van den Bosch F, et al. Safety and efficacy of upadacitinib in patients with rheumatoid arthritis and inadequate response to conventional synthetic disease-modifying anti-rheumatic drugs (SELECT-NEXT): a randomised, double-blind, placebo-controlled phase 3 trial. Lancet 2018;391:2503-12.

12 Fleischmann R, Pangan AL, Song I-H, et al. Upadacitinib versus placebo or adalimumab in patients with rheumatoid arthritis and an inadequate response to methotrexate: results of a phase III, double-blind, randomized controlled trial. Arthritis Rheumatol 2019;71:1788-800.

13 Genovese MC, Fleischmann R, Combe B, et al. Safety and efficacy of upadacitinib in patients with active rheumatoid arthritis refractory to biologic disease-modifying anti-rheumatic drugs (select-beyond): a double-blind, randomised controlled phase 3 trial. Lancet 2018;391:2513-24.

14 Smolen JS, Pangan AL, Emery P, et al. Upadacitinib as monotherapy in patients with active rheumatoid arthritis and inadequate response to methotrexate (select-monotherapy): a randomised, placebocontrolled, double-blind phase 3 study. Lancet 2019;393:2303-11.

15 van Vollenhoven R, Takeuchi T, Pangan AL, et al. Efficacy and safety of upadacitinib monotherapy in methotrexate-naive patients with moderately to severely active rheumatoid arthritis (SELECT-EARLY): a randomized, double-blind, active-comparator, multi-center, multicountry trial. Arthritis Rheumatol 2020;72:1607-20.

16 Mclnnes IB, Anderson JK, Magrey M, et al. Trial of upadacitinib and adalimumab for psoriatic arthritis. N Engl J Med 2021;384:1227-39.

17 Krishnan E, Sokka T, Häkkinen A, et al. Normative values for the health assessment questionnaire disability index: benchmarking disability in the general population. Arthritis Rheum 2004;50:953-60.

18 Cohen SB, van Vollenhoven RF, Winthrop KL, et al. Safety profile of upadacitinib in rheumatoid arthritis: integrated analysis from the select phase III clinical programme. Ann Rheum Dis 2020;80:304-11. 
Correction: Upadacitinib in patients with psoriatic arthritis and an inadequate response to non-biological therapy: 56-week data from the phase 3 SELECT-PSA 1 study

McInnes IB, Kato K, Magrey M, et al. Upadacitinib in patients with psoriatic arthritis and an inadequate response to non-biological therapy: 56-week data from the phase 3 SELECT-PsA 1 study. RMD Open 2021;7:e001838. doi: 10.1136/rmdopen-2021-001838

In this article, the author name 'Frank Behrens' was incorrectly spelt as 'Franck Behrens'. This has now been corrected online.

Open access This is an open access article distributed in accordance with the Creative Commons Attribution Non Commercial (CC BY-NC 4.0) license, which permits others to distribute, remix, adapt, build upon this work noncommercially, and license their derivative works on different terms, provided the original work is properly cited, appropriate credit is given, any changes made indicated, and the use is non-commercial. See: http://creativecommons. org/licenses/by-nc/4.0/.

(c) Author(s) (or their employer(s)) 2021. Re-use permitted under CC BY-NC. No commercial re-use. See rights and permissions. Published by BMJ.

RMD Open 2021;7:e001838corr1. doi:10.1136/rmdopen-2021-001838corr1

Check for updates 